\begin{tabular}{|c|c|c|}
\hline Beitr. Ent. & Berlin & ISSN 0005-805X \\
\hline $49(1999) 1$ & S. $97-105$ & 6.04 .1999 \\
\hline
\end{tabular}

\title{
Eine neue Art der Gattung Adinopsis CAMERon aus dem Baltischen Bernstein
}

\section{(Coleoptera: Staphylinidae, Aleocharinae, Deinopsini)}

Mit 10 Figuren

LOTHAR ZERCHE

\section{Zusammenfassung}

Die erste fossile Art aus der Tribus Deinopsini, Adinopsis ${ }^{\dagger}$ groehni sp. n., aus dem tertiären Baltischen Bernstein [ca. 35 Millionen Jahre] wird beschrieben und mit den rezenten Arten verglichen. Die fossile Art ähnelt in zwei wichtigen Antennen-Merkmalen der australischen Art Adinopsis australis (FAUVEL). Diese Ähnlichkeit beruht aber nur auf Symplesiomorphien. Adinopsis ${ }^{\dagger}$ groehni sp. n. ist eine stammesgeschichtlich isolierte Art, für die deshalb eine neue Artengruppe, die Adinopsis ${ }^{\dagger}$ groehni-Gruppe, errichtet wird, die am Beginn der "World checklist" von KLMASZEWSKI \& JANSEN (1994) einzuordnen ist, gefolgt von der Adinopsis australis-Gruppe, die aus phylogenetischen Gründen anders plaziert werden muß.

\section{Summary}

The first fossil species of the tribe Deinopsini, Adinopsis ${ }^{\dagger}$ groehni sp. n., from Tertiary Baltic amber [about 35 million years] is described and compared with the recent species. The fossil species is similar to Adinopsis australis (FAUVEL) in two important antennal characters. But this similarity is based on symplesiomorphic characters only. Adinopsis ${ }^{\dagger}$ groehni $\mathrm{sp} . \mathrm{n}$. is a phylogenetically isolated species, for which, therefore, a new species group, the Adinopsis ${ }^{\dagger}$ groehni-group, is established. This group must be placed at the beginning of the world checklist by KLIMASZEWSKI \& JANSEN (1994) followed by the Adinopsis australis-group whose systematic position must be altered for phylogenetic reasons.

\section{Einleitung}

Unter zahlreichen Inklusen aus verschiedenen Unterfamilien der Familie Staphylinidae, die mir zur Determination vorgelegt wurden, fand ich ein Tier, das zur Gattung Adinopsis gehört. Es war unproblematisch, dieses Exemplar als Vertreter einer noch unbeschriebenen Art zu identifizieren, weil alle Arten der Gattung Adinopsis in jüngerer Zeit nach modernen Kriterien revidiert oder neu beschrieben worden sind (KLIMASZEWSKI 1979, 1980, 1982, 1985, 1991, KLIMASZEWSKI \& GENIER 1985, KLIMASZEWSKI \& FRANK 1992, KLIMASZEWSKI \& JANSEN 1994, PACE 1987, UHLIG 1995, UHLIG \& KLIMASZEWSKI 1995, JANÁK 1996). 
Zur Gattung Adinopsis CAMERON, 1919 gehören 23 rezente Arten, die überwiegend tropisch verbreitet sind und sich auf die folgenden zoogeographischen Regionen verteilen: Afrotropis (10 Arten), Madagassische Subregion (2 Arten), Neotropis (4 Arten), Orientalis (3 Arten), Australis (1 Art), Nearktis (3 Arten).

\section{Danksagung}

Sehr herzlich danke ich Herrn CARSTEN GRÖHN, Glinde, der mir seine interessanten Staphylinidae-Inklusen vorgelegt hat, den "Adinopsis-Stein" bearbeitet und die Fotos angefertigt hat und dem ich auch den Holotypus für die Sammlung des DEI verdanke. Frau BIANKa KATNIG, Eberswalde, danke ich für die Anfertigung der Zeichnungen nach dem Original. MANFRED UHLIG, Berlin, und CARSTEN GRÖHN bin ich zu Dank für die Durchsicht des Manuskripts verpflichtet.

\section{Adinopsis 'ंgroehni sp. n.}

Fig. 1-10

\section{Typenmaterial}

Holotypus, wahrscheinlich ơ: Inkluse in Baltischem Bernstein $\backslash$ Holotypus Adinopsis ${ }^{\dagger}$ groehni ZERCHE (DEI).

Locus typicus: mit großer Wahrscheinlichkeit Palmnicken-Königsberg, auf jeden Fall aus dieser Gegend (GRÖHN i. 1.).

Stratum typicum: Tertiär, wahrscheinlich Unter-Oligozän [ca. 35 Millionen Jahre (nach WErTSCHAT 1996)].

\section{Bernstein-Material, Bearbeitung, Syninklusen (GRöHN i. 1.)}

Die hell-gelb-klare Bernsteinschlaube wog vor der Bearbeitung ca. 3 g. Als Syninklusen neben Adinopsis waren enthalten: 2 Staphylinidae, Paederinae; 1 Zweigspitze von Thuja; 1 Chironomidae, 1 kleine unbestimmbare Larve; wenige Sternhaare.

Um Adinopsis besser einsehen zu können, mußte das Bernsteinstück auf weniger als $1 \mathrm{~g}$ beschliffen werden. Als Syninkluse blieb nur 1 Staphylinidae, Paederinae, erhalten.

Die Endbearbeitung erfolgte mit 4000er Naßschleifpapier und Politur an der Schwabbelscheibe. Zur Konservierung wurde der Inklusenstein mit Polyurethan-Lack beschichtet.

\section{Beschreibung}

Maße des Holotypus [in mm]: Körperlänge 2,8; Kopfbreite 0,53; Länge der Antennen ca. 0,84; Pronotumlänge 0,53; Pronotumbreite 0,78; Nahtlänge 0,39 ; Elytrenbreite ca. 0,84 ; Länge der Abdominalsegmente III 0,22; IV 0,21; V 0,22; VI 0,22; VII 0,38; VIII 0,32.

Farbe [soweit wegen der teilweisen Verlumung ${ }^{1}$ erkennbar] schwarz. Punktur sehr fein. Behaarung sehr fein und anliegend, auf dem Sternit VIII etwas länger und weniger dicht als auf den vorderen Sterniten. Die Hinterränder der Segmente III bis VI (Tergite, Paratergite und Sternite) mit dichten Reihen kräftiger kammförmig angeordneter Setae besetzt. Tergit und Sternit VIII am Hinterrand mit stärkerer, längerer und fast palisadenartig angeordneter borstenförmiger Behaarung [Wahrscheinlich sind diese Terminalhaare des Sternits VIII apikal kurz gegabelt; dies kann jedoch wegen der bei stärkerer Vergrößerung zu geringen Schärfe nicht eindeutig beurteilt werden (100x)]; diese Behaanung am Tergit in der Mitte der Einbuchtung weniger dicht stehend. Sternit VIII auch im hinteren Teil, vor allem seitlich, schräg abstehend behaart.

\footnotetext{
${ }^{1}$ Verlumung: Die weißliche, undurchsichtige Trübung des Bernsteins ist entstanden, wenn Inklusen lebend bzw. feucht ins Harz gerieten.
} 
Kopf breit [aber stark verlumt und durch eine zweite Inkluse teilweise verdeckt, nur die Breite erkennbar, sonst kaum sichtbar]. Antennen robust, nicht fadenförmig, alle Segmente zwar länger als breit [Segmente \pm verlumt, deshalb nicht völlig klar erkennbar], die Segmente VII bis X aber nicht doppelt so lang wie breit. Segment XI annähernd kahnförmig, nach vorn und hinten verengt, ohne apikale Abschnürung (Papilla), etwa 2-2,5mal so lang wie breit.

Pronotum stark quer (Breite : Länge 1,5). Vorderrand flach konvex; Vorderwinkel breit abgerundet [aber nicht gut sichtbar]. Seitenränder fast gleichmäßig gebogen, ihre breiteste Stelle etwa am Beginn des letzten Drittels. Hinterrand in der Mitte breit konvex, neben den Hinterwinkeln sehr deutlich ausgeschweift (konkav). Hinterwinkel scharf, etwa rechtwinklig [Unterseite des Vorderkörpers weitgehend verlumt, nicht erkennbar].

Elytren kürzer als das Pronotum, Seiten fast geradlinig nach hinten verengt. Hinterrand zur Naht breit konkav, neben den Hinterwinkeln deutlich ausgerandet.

Abdomen von Segment III bis VIII nahezu gleichmäßig verengt. Hinterrand des Tergits VII mit schmalem Fransensaum. Hinterrand des Tergits VIII breit stumpfwinklig eingebuchtet. Hinterrand des Sternits VII in der Mitte flach stumpfwinklig vorgezogen. Sternit VIII mit einem langgestreckten flachen Eindruck, der von der flachen, aber deutlichen Einbuchtung des Hinterrandes ausgeht [aber insgesamt nicht klar erkennbar].

Beine in der für die Gattung charakteristischen Form [aber weitgehend verlumt und nur in Umrissen erkennbar, nur die Tarsen besser sichtbar]. Tarsenformel 2, 2, 2. Segment I der Tarsen sehr gestreckt und deutlich länger als das Segment II. Beborstung der Schienen und Ausbildung der Klauensegmente in der für die Deinopsini charakteristischen Weise.

\section{Differentialdiagnose}

Die neue fossile Art ist sehr leicht von allen rezenten Arten zu unterscheiden. Bei keiner der 23 rezenten Arten ist der Hinterrand des Sternits VIII eingebuchtet (konkav), dieser ist entweder gerade abgestutzt oder flach gerundet (konvex). Von Adinopsis australis, mit der sie im Bau der Antennen übereinstimmt, ist sie durch die völlig anderere Bildung des Hinterrandes des Tergits VIII zu unterscheiden. Dieser ist bei ${ }^{\dagger}$ groehni breit und flach, bei australis dagegen schmal und tief [Deinopsiswähnlich] eingebuchtet [Abbildung des Tergits VIII (KLIMASZEWSKI 1979: Fig. 193)]. Von den restlichen 22 rezenten Arten unterscheidet sich Adinopsis ${ }^{\dagger}$ groehni durch den Bau der Antennen auf den ersten Blick, weil diese nicht fadenförmig sind und weil am Segment XI eine apikale Papille fehlt.

\section{Verwandischaftliche Stellung}

Die fossile Art wird der Gattung Adinopsis zugeordnet, weil sie wie die rezenten Arten zweisegmentige Tarsen mit den für die Gattung charakteristischen Proportionen hat: erstes Tarsenglied gestreckt, bei allen Beinen deutlich länger als das Klauenglied [bei Deinopsis Tarsen dreisegmentig und Glieder I und II zusammen kürzer als das Klauenglied]. Für die Zuordnung zur Gattung Adinopsis spricht auch der Bau des Abdominaltergits VIII. Sein Hinterrand ist breit und nur flach eingebuchtet [bei Deinopsis diese Einbuchtung in beiden Geschlechtern schmal und tief]. Eine apikale Abschnürung am Antennensegment XI fehlt bei Adinopsis ${ }^{\dagger}$ groehni sp. n.; sie fehlt aber nach KLIMASZEWSKI (1979: Abbildung der Antenne Fig. 201) auch bei der rezenten Art Adinopsis australis (FAUVEL, 1878) aus Victoria, Australien.

Die neue fossile Art steht verwandtschaftlich isoliert da. Alle Arten, deren Antennen fadenförmig gebaut sind (Synapomorphie), und die über eine apikale Papille am Segment XI verfügen (Synapomorphie), bilden ein Monophylum. Adinopsis australis und Adinopsis ${ }^{\dagger}$ groehni stehen außerhalb dieses Monophylums, da sie in diesen beiden Antennen-Merkmalen jeweils die plesiomorphe Alternative aufweisen. Die beiden Arten australis und 'tgroehni sind nicht näher miteinander verwandt. Dafür spricht die völlig unterschiedliche Ausbildung der Hinterränder von Tergit und Sternit VIII. Thre Ähnlichkeit benuht nur auf Symplesiomorphien. 
Die flache Einbuchtung des Hinterrandes des Sternits VIII ist als Autapomorphie von Adinopsis ${ }^{\dagger}$ groehni zu werten, da diese Sonderbildung nur bei ihr auftritt.

Zur Einordnung der neuen Art in die "World checklist" von KLIMASZEWSKI \& JANSEN (1994) wird hiermit eine neue Artengruppe, die Adinopsis ${ }^{\dagger}$ groehni-Gruppe, gebildet, die aus verwandtschaftlichen Gründen den anderen Artengruppen vorangestellt wird (Artengruppe Nr. 1). Die Adinopsis australis-Gruppe, die in der "World checklist" von KLIMASZEWSKI \& JANSEN (1994) als "polyphyletischer Fremdkörper" inmitten eines Monophylums untergebracht worden ist, sollte nach der Adinopsis ${ }^{\dagger}$ groehni-Gruppe an zweiter Stelle eingereiht werden (Artengruppe Nr. 2).

Alle folgenden Gruppen bilden dann ein Monophylum setis mutabilis, ausgenommen die beiden relativ am stärksten abgeleiteten nearktischen Arten, die von KLIMASZEWSKI \& JANSEN (1994) in die Untergattung Condylopennis gestellt wurden und in der Adinopsis bicornis-Gruppe am Ende der "World checklist" korrekt plaziert scheinen. Ihr Untergattungsname Condylopennis ist jedoch ein nomen nudum, da zwei Arten in die Untergattung gestellt wurden und die Autoren es versäumt haben, eine Typusart zu designieren.

\section{Stammesgeschichtliches Alter}

Das Alter des Baltischen Bernsteins wird von WEITSCHAT (1996) mit etwa 35 Millionen Jahren angegeben, dessen Argumentation ich im vorstehenden Text gefolgt bin.

RÖSCHMANN (1994) zitiert für das absolute Alter ANDREE (1937) mit 35-40 Millionen Jahren.

\section{Derivatio nominis}

Die neue Art ist Herrn CARSTEN GRÖHN, Glinde, gewidmet.

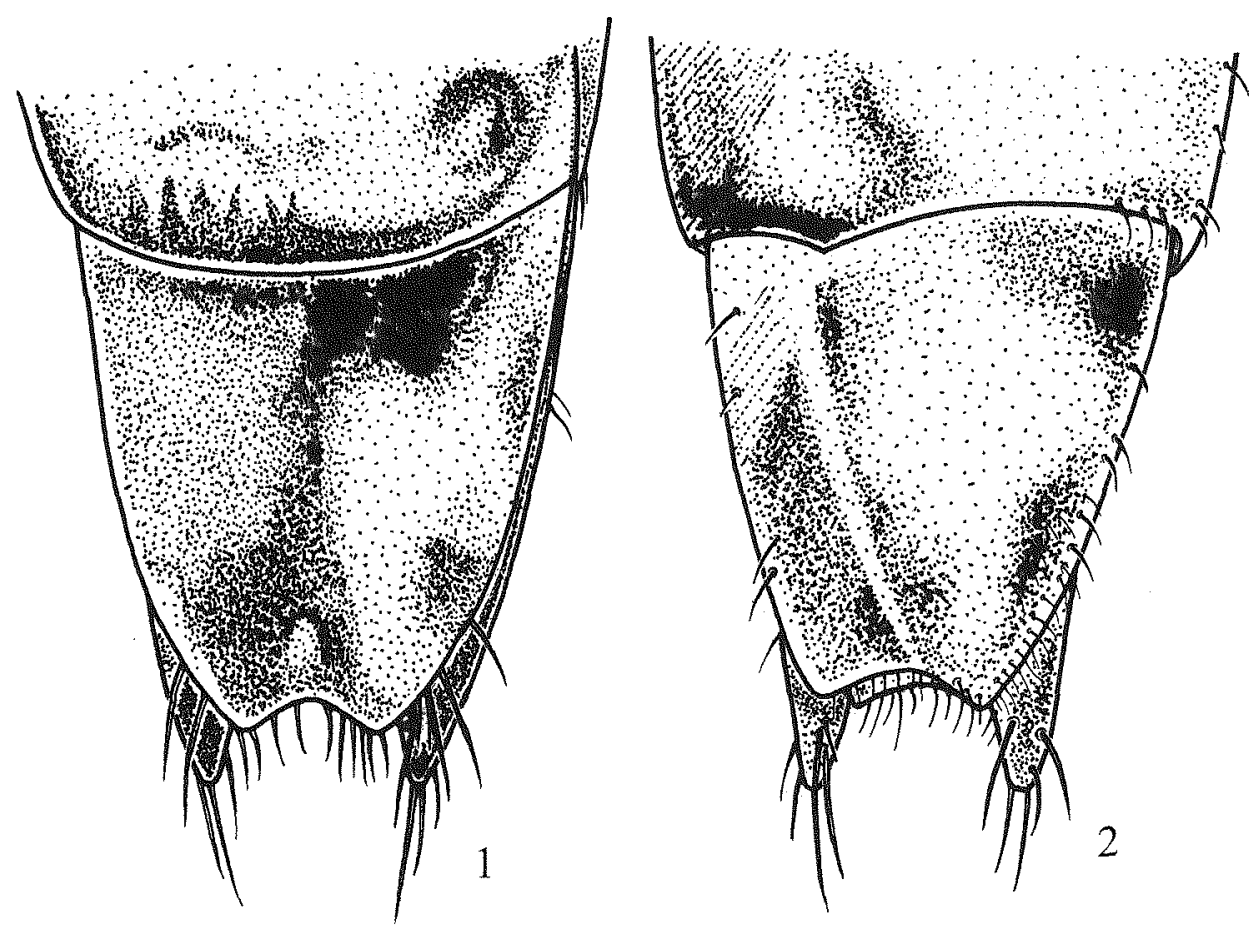

Fig. 1-2. Adinopsis ${ }^{\dagger}$ groehni sp. n.: Fig. 1. Abdomenspitze, dorsal. - Fig. 2. Abdomenspitze, ventral. 

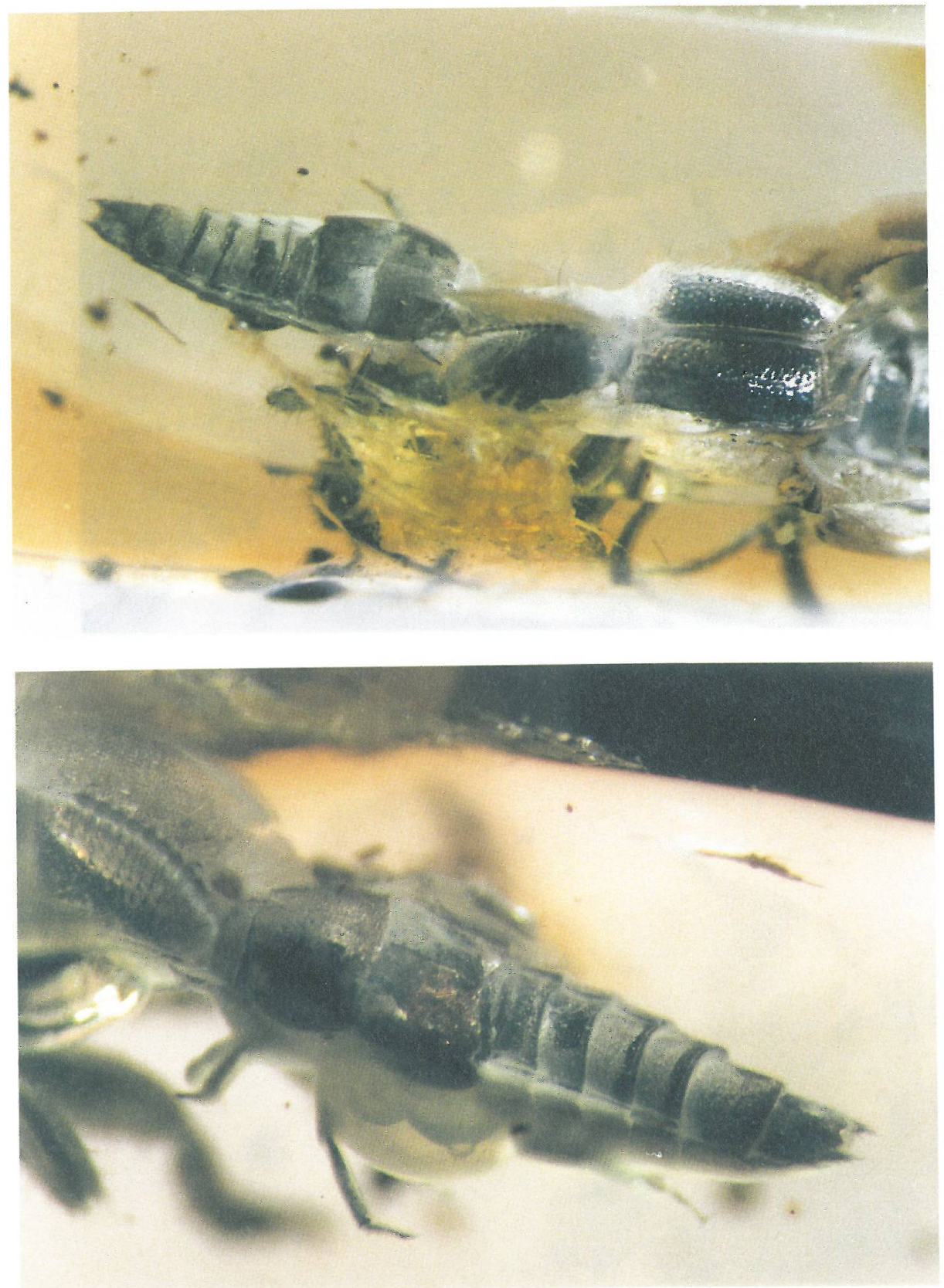

Fig. 3-4: Adinopsis ${ }^{\dagger}$ groehni sp. n. - Habitus, dorsal. 

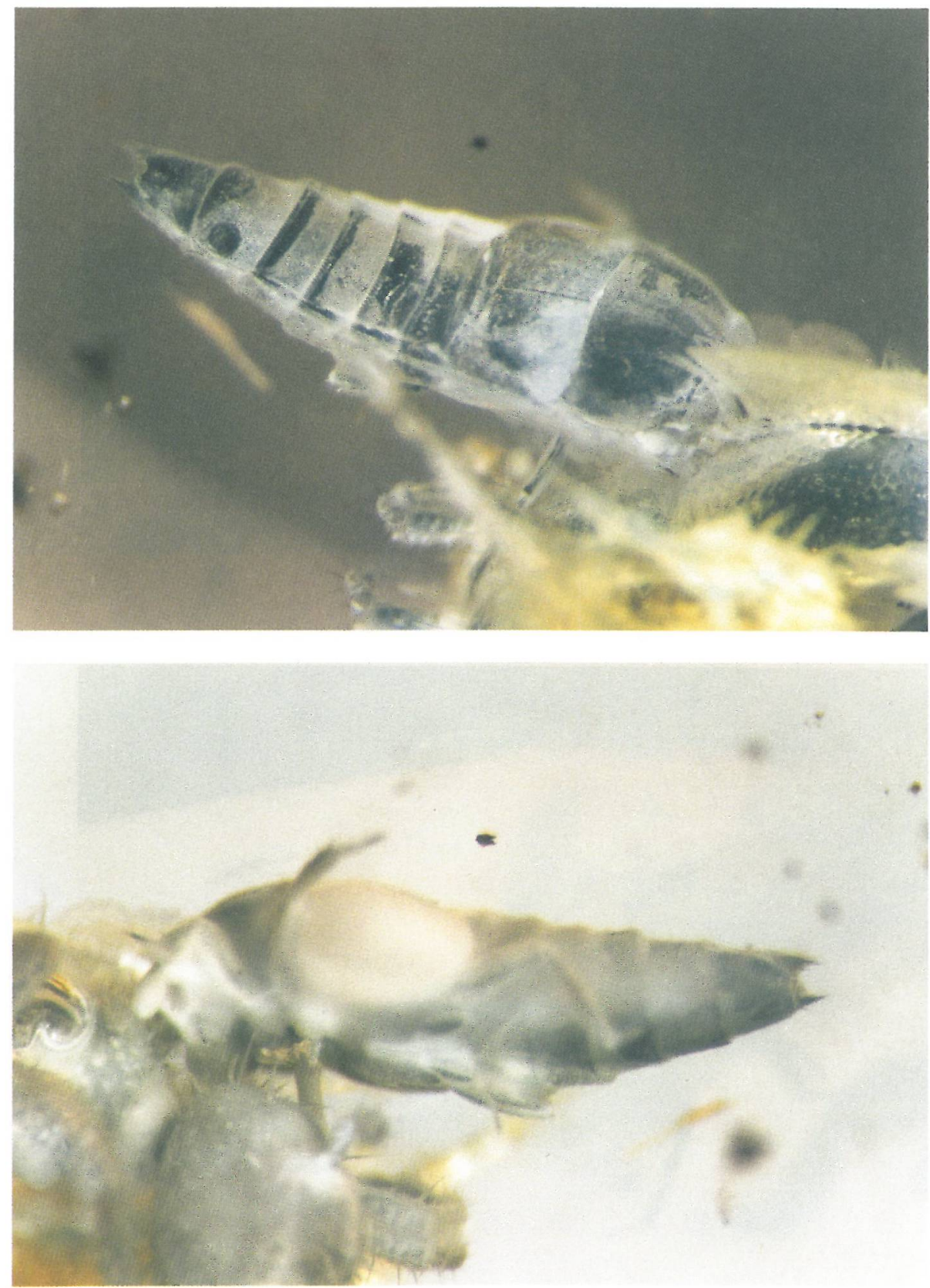

Fig. 5. Lage der Inklusen im Bernstein - Adinopsis ${ }^{\dagger}$ groehni sp. n. (links) - Paederinae (rechts). Fig. 6. Adinopsis ${ }^{\dagger}$ groehni sp. n. - Habitus, ventral. 

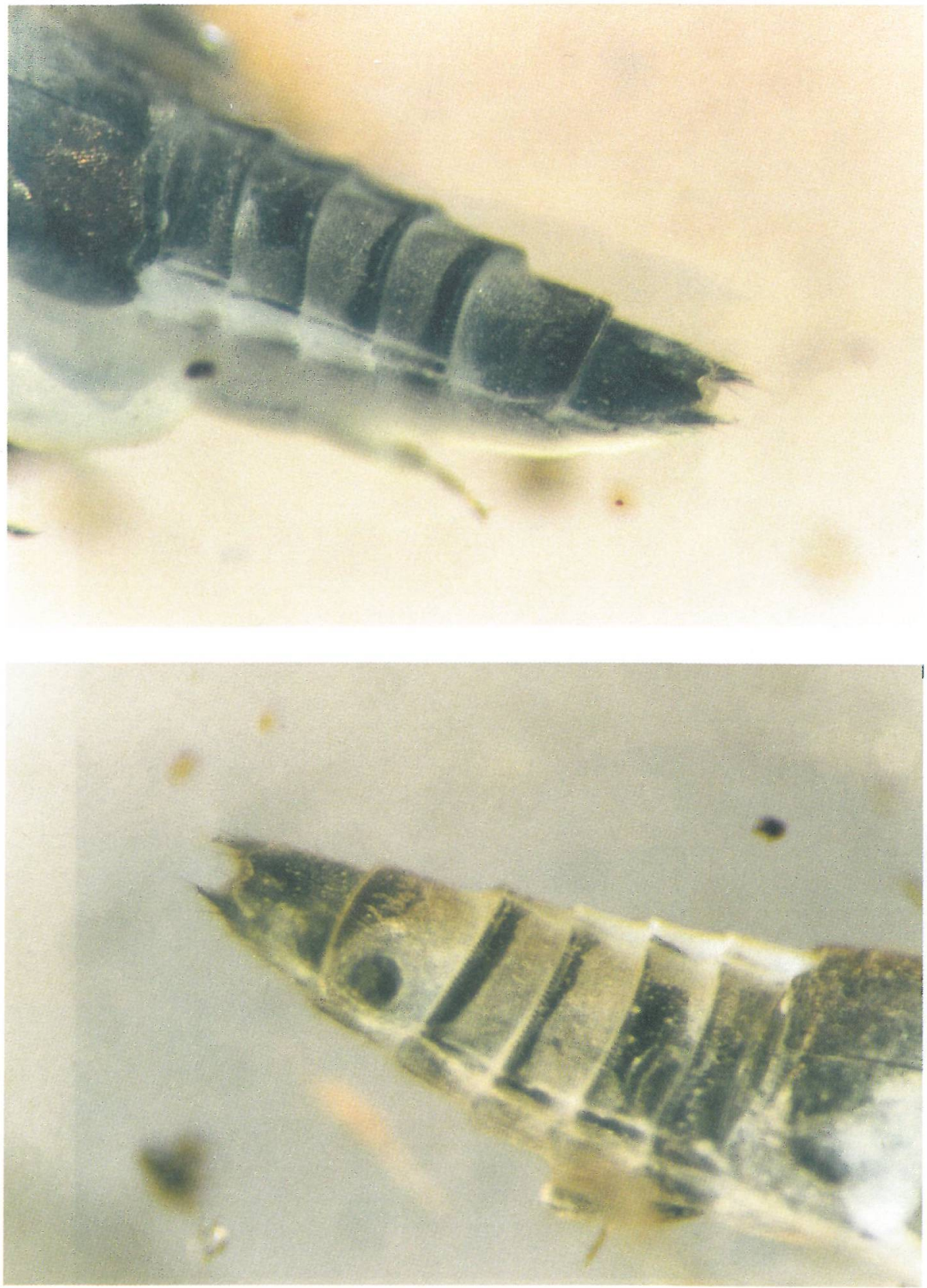

Fig. 7-8. Adinopsis ${ }^{\dagger}$ groehni sp. n.: Abdomen, dorsal. 


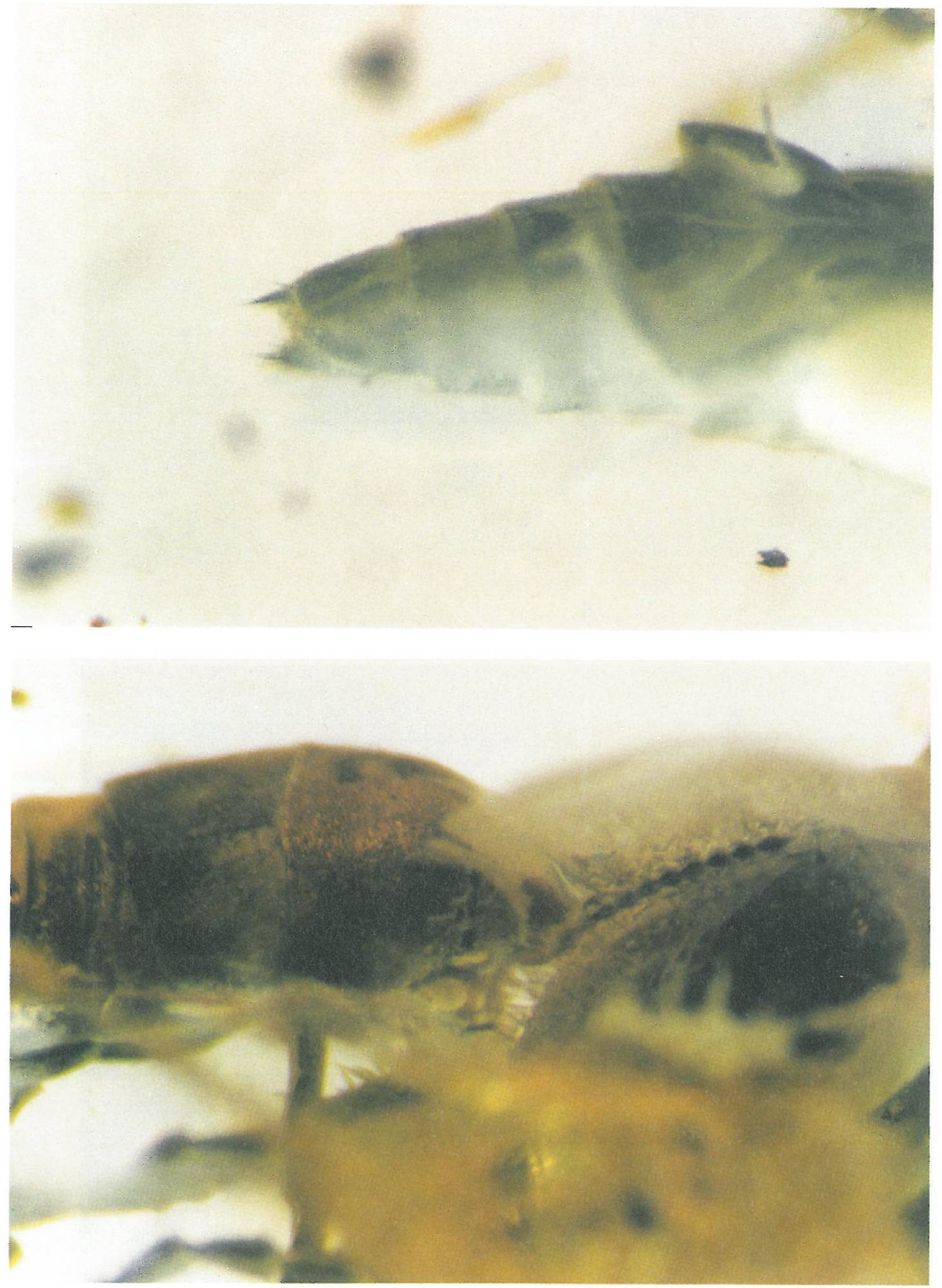

Fig. 9-10. Adinopsis ${ }^{\dagger}$ groehni sp. n.: Fig. 9. Abdomen, ventral. - Fig. 10. Vorderkörper mit rechter Antenne, dorsolateral. 


\title{
Literatur
}

JANÁK, J. 1996: Zwei neue Arten der Gattung Adinopsis aus Madagaskar (Coleoptera, Staphylinidae, Aleocharinae, Deinopsini). - Entomofauna, Ansfelden 17(19): 325-336.

KIIMASZEWSKI, J. 1979: A revision of the Gymnusini and Deinopsini of the world. Coleoptera: Staphylinidae, Aleocharinae. - Monograph 25, Agriculture Canada, Ottawa: $169 \mathrm{~S}$.

KIIMASZEWSKI, J. 1980: Two new species of Deinopsini from the Afrotropical and Nearctic regions, with notes on two other species of this tribe (Coleoptera, Staphylinidae). - Polskie Pismo Ent., Warszawa; Wroclaw 50: 109-120.

KLIMASZEWSKI, J. 1982: A revision of the Gymnusini and Deinopsini of the world (Coleoptera: Staphylinidae). Supplementum 2. - Can. Ent., Ottawa 114: 317-335.

KIMMASZEWSKI, J. 1985: Revision of the Gymnusini and Deinopsini of the world (Coleoptera: Staphylinidae). Supplement 4. New distribution data and description of female Adinopsis bicomis. - Ent. News, Philadelphia 96: 142-144.

KIIMASZEWSKI, J. \& GÉNIER, F. 1985: A revision of the Gymnusini and Deinopsini of the world (Coleoptera: Staphylinidae). Supplementum 3. - Coleopts Bull., Washington D. C. 39: 60-66.

KIIMASzEWSKI, J. 1991: First record of the genus Adinopsis CAMERon from South Africa, with the description of a new species (Coleoptera: Staphylinidae: Aleocharinae). - Annals of the Transvaal Museum 35(13): 205-207.

KIIMASZEWSKI, J. \& FRANK, J. H. 1992: New distributional data for New World Gymnusini and Deinopsini, with description of a new species (Coleoptera: Staphylinidae, Aleocharinae). Supplement 5. - Coleopts Bull., Washington D. C. 46(3): 242-249.

KIIMASZEWSKI, J. \& JANSEN, R. 1994: Description of a new Afrotropical species of Adinopsis CAMERON 1919 with notes on some Neotropical and/or Nearctic species (Coleoptera, Staphylinidae, Aleocharinae). Tropical Zoology, Firenze 7: 325-332.

PACE, R. 1987: Staphylinidae dell'Himalaya Nepalese. Aleocharinae raccolte dal Prof. Dr. J. MARTENS (Insecta: Coleoptera). - Courier Forschungsinstitut Senckenberg, Frankfurt am Main 93: 383-441.

RösCHMANN, F. 1994: Die Sciariden des Baltischen und des Sächsischen Bernsteins (Insecta, Diptera, Sciaridae). - Dissertation, Math.-Nat. Fakultät der Emst-Moritz-Arndt-Universität Greifswald: $183 \mathrm{~S}$.

UHLIG, M. 1995: Adinopsis klimaszewskii sp. n. from the Popa Falls in Northern Namibia (Coleoptera, Staphylinidae, Aleocharinae). - Mitt. Zool. Mus. Berl. 71(2): 293-295.

UHLIG, M. \& KLMMASZEWSKI, J. 1995: A review of the African Adinopsis species with description of two new species from Northern Namibia (Coleoptera, Staphylinidae, Aleocharinae). - Mitt. Zool. Mus. Berl. 71(2): 297-309.

Werrschat, W. 1996: Bernstein in der Deutschen Bucht und in Jütland auf 3., 4., 5. oder 6. Lagerstätte. - In: Ganzelewski, M. \& SlotTA, R. (Hrsg.) - Tränen der Götter. Katalog zur Ausstellung des Deutschen Bergbau-Museums Bochum: 77-82.

\author{
Anschrift des Verfassers: \\ Dr. LOTHAR ZERCHE \\ Deutsches Entomologisches Institut \\ Schicklerstraße 5 \\ D- 16205 Eberswalde, Deutschland \\ Zerche@dei-eberswalde.de
}

